\title{
Three dimensional echocardiography documents haemodynamic improvement by biventricular pacing in patients with severe heart failure
}

\author{
W Y Kim, P Søgaard, P T Mortensen, H Kjærulf Jensen, A Kirstein Pedersen, \\ B Ø Kristensen, H Egeblad
}

\begin{abstract}
Objectives-To quantify the short term haemodynamic effects of biventricular pacing in patients with heart failure and left bundle branch block by using three dimensional echocardiography.

Design-Three dimensional echocardiography was performed in 15 consecutive heart failure patients (New York Heart Association functional class III or IV) with an implanted biventricular pacing system. Six minute walk tests were performed to investigate the effect of biventricular pacing on exercise capacity. Data were acquired at sinus rhythm and after short term (2-7 days) biventricular pacing.

Results-Compared with baseline values, biventricular pacing significantly reduced left ventricular end diastolic volume (EDV) by mean (SD) $4.0(5.1) \%(\mathrm{p}<0.01)$ and end systolic volume (ESV) by $5.6(6.4) \%(\mathrm{p}<0.02)$. Mitral regurgitant fraction was significantly reduced by $11(12.1) \%(\mathrm{p}<0.003)$ and forward stroke volume (FSV) increased by $13.9(18.6) \%(\mathrm{p}<0.02)$. Exercise capacity was significantly improved with biventricular pacing by 48.4 (43.3)\% $(\mathrm{p}<0.00001)$. Regression analyses showed that the percentage increase in FSV independently predicted percentage improvement in walking distance $\left(r^{2}=0.73, \mathrm{p}<0.0002\right)$. Both basal QRS duration and QRS narrowing predicted pacing efficacy, showing a significant correlation with $\% \Delta \mathrm{EDV}, \% \Delta \mathrm{ESV}$, and $\% \Delta \mathrm{FSV}$.

Conclusions-In five of 15 consecutive patients with heart failure and left bundle branch block, biventricular pacing induced a more than $15 \%$ increase in FSV, which predicted a more than $25 \%$ increase in walking distance and was accompanied by an immediate reduction in left ventricular chamber size and mitral regurgitation.

(Heart 2001;85:514-520)
\end{abstract}

Keywords: heart failure; left ventricular volume; pacing; three dimensional echocardiography

Pacing as an adjunctive treatment in selected patients with severe heart failure and left bundle branch block is being investigated in clinical trials. Initial research was focused on dual chamber pacing with shortening of the atrioventricular interval to optimise ventricular filling and limit presystolic mitral regurgitation. ${ }^{1-3}$ More recently, simultaneous biventricular pacing to restore systolic contractile synchrony has been investigated. Thus, in heart failure patients both biventricular and single site left ventricular (LV) free wall pacing have been shown to enhance femoral systolic pressure acutely while lowering pulmonary wedge pressure and $\mathrm{V}$ wave amplitude. ${ }^{4}$ Similar results have been reported by other investigators showing significantly increased cardiac index and decreased pulmonary wedge pressure. ${ }^{5}$ In addition, pressure-volume analysis revealed that biventricular pacing acutely enhanced contractile function in heart failure patients with intraventricular conduction delay. ${ }^{6}$ Thus, improved contractile function, reduced mitral regurgitation, and optimised LV filling have all been proposed as beneficial effects of biventricular pacing. However, the relative importance of the proposed mechanisms is unclear and a direct correlation to the patient's clinical status is missing. Thus, a non-invasive protocol is needed to evaluate pacing related haemodynamic changes outside the catheterisation laboratory.
Recent advances in three dimensional (3D) echocardiography using tissue harmonic imaging allow us to measure LV volumes with excellent reproducibility. ${ }^{7}$ In addition, a novel 3D colour Doppler technique has been shown to quantify volumetric flow in the large vessels accurately. ${ }^{8-11}$ No previous study has quantified the impact of biventricular pacing on forward stroke volume and mitral regurgitation or the possible changes in LV chamber size. Neither have pacing induced haemodynamic changes been correlated directly with exercise capacity. To address these issues, we performed a 3D echocardiographic study of short term biventricular pacing effects in patients with dilated cardiomyopathy. We investigated the following hypotheses: firstly, that biventricular pacing reduces functional systolic mitral regurgitation and increases cardiac output in patients with heart failure; secondly, that LV volumes are reduced because of electrical cardiac resynchronisation; and lastly, that a favourable haemodynamic response to biventricular pacing is reflected by improved exercise capacity in patients with dilated cardiomyopathy.

\section{Methods}

STUDY GROUP

Fifteen consecutive male patients in sinus rhythm with bundle branch block (QRS duration > $120 \mathrm{~ms}$ measured as the maximum 
Table 1 Baseline clinical characteristics of patient group

\begin{tabular}{llllllll}
\hline Patient & Age (years) & EF (\%) & $\begin{array}{l}\text { HR } \\
\text { (beats/min) }\end{array}$ & QRS (ms) & $\begin{array}{l}\text { NYHA } \\
\text { class }\end{array}$ & Type & LBBB \\
\hline 1 & 69 & 29 & 83 & 200 & IV & ICM & + \\
2 & 75 & 32 & 72 & 200 & IV & ICM & (RBBB) \\
3 & 63 & 21 & 84 & 178 & III & ICM & + \\
4 & 42 & 18 & 98 & 240 & IV & IDC & + \\
5 & 67 & 26 & 64 & 165 & IV & ICM & + \\
6 & 59 & 27 & 96 & 161 & III & AO & + \\
7 & 57 & 33 & 71 & 196 & III & ICM & + \\
8 & 64 & 21 & 85 & 200 & III & ICM & + \\
9 & 76 & 26 & 53 & 160 & III & IDC & + \\
10 & 66 & 16 & 88 & 180 & III & ICM & + \\
11 & 52 & 26 & 82 & 180 & IV & ICM & + \\
12 & 66 & 32 & 92 & 180 & IV & ICM & + \\
13 & 72 & 32 & 74 & 165 & III & ICM & + \\
14 & 66 & 35 & 56 & 145 & III & IDC & + \\
15 & 59 & 21 & 91 & 180 & III & ICM & + \\
Mean (SD) & $63.5(8.9)$ & $26.4(5.9)$ & $79.3(13.9)$ & $182(23)$ & & & \\
& & & & & &
\end{tabular}

$\mathrm{AO}$, aortic valve replacement caused by stenosis; $\mathrm{EF}$, ejection fraction; $\mathrm{HR}$, sinus rhythm heart rate; ICM, ischaemic cardiomyopathy; IDC, idiopathic dilated cardiomyopathy; LBBB, left bundle branch block; NYHA, New York Heart Association; QRS, mean duration; RBBB right bundle-branch block.

width from a 12 lead surface ECG with a paper speed of $50 \mathrm{~mm} / \mathrm{s}$ ) and severe heart failure symptoms (New York Heart Association functional class III or IV) despite contemporary medical treatment were enrolled in the study. The study protocol included pacemaker implantation and 3D echocardiographic examination. Written informed consent was obtained from each patient in accordance with the regional ethical committee on human research. Patients were all in a stable haemodynamic condition at the time of their inclusion into the study. The anticongestive medication consisted of diuretics, angiotensin converting enzyme inhibitors, $\beta$ blockers, digoxin, and aldosterone antagonist. Table 1 provides baseline clinical characteristics.

PACING PROTOCOL

All patients had a permanent left sided biventricular pacemaker system implanted. Using either subclavian vein puncture or cephalic vein putdown, three transvenous pacing leads were implanted, one in the high right atrium, one in the septal region of the right ventricle, and one in the coronary sinus system (left ventricle). Active fixation leads (Sweet Picotip, CPI, St Paul, Minnesota, USA) were used for the right atrium and right ventricle. To pace the left ventricle, either a standard pacing lead (Slimtine, Vitatron, Dieren, The Netherlands) or a specially designed coronary sinus lead (2187 or 10512, Medtronic, Minneapolis, Minnesota, USA) was guided into the coronary sinus system. The tip electrode was then placed in a stable position in a side branch, preferably in the middle part of the lateral or posterolateral region.

For optimal LV resynchronisation during biventricular pacing, the right ventricular and LV pacing leads were placed so that the sensed local intracardiac activation during sinus rhythm was measured as early as possible for the right ventricular lead and as late as possible for the LV lead. ${ }^{5}$ Thereby the widest possible electrical and anatomical separation was obtained between the two ventricular pacing leads, ensuring maximal "normalisation" of QRS duration on surface ECG during biventricular pacing.
The pacing leads were connected to a dual chamber biventricular pacemaker (InSync, Medtronic) with simultaneous pacing of the two ventricular leads. The biventricular pacemaker was programmed in DDD mode and for each patient the atrioventricular interval was adjusted to maximise mitral inflow duration with the aid of pulsed Doppler recordings. Atrial rates were kept at identical values during the comparative 3D echocardiographic measurements with and without biventricular pacing.

ECHOCARDIOGRAPHIC PROTOCOL

Ten of the 15 subjects underwent measurement of LV volumes and volumetric flows by $3 \mathrm{D}$ echocardiography on the day before pacemaker implantation and 2-7 days (mean 2.9 days) afterwards. Five of the patients were investigated on the day after implantation with biventricular pacing on and off. These measurements were first taken with biventricular pacing on and then repeated after pacing had been off for approximately half an hour.

Transthoracic 3D echocardiography was performed with the subjects in the left lateral decubitus position (Vingmed SystemFive, GE Vingmed Ultrasound, Horten, Norway). LV volumes and volumetric flows through the LV outflow tract and mitral valve were acquired by coaxial (rotational) scanning from an apical position. ${ }^{12}$ The initial apical image of the LV long axis was selected arbitrarily to ensure absence of sampling bias, and six sections were acquired with a $30^{\circ}$ interval by automated rotation of the transducer. LV volume recordings were made in tissue harmonic mode to improve endocardial border detection using a transducer frequency of 1.6 or $1.7 \mathrm{MHz}$, depending on the acoustic properties of the subjects.

Volumetric flows through the LV outflow tract and mitral valve were measured by $3 \mathrm{D}$ colour Doppler by rotational data acquisition similar to the LV volume measurements. The colour flow data were recorded at $2.5 \mathrm{MHZ}$ and low velocity rejection was set to $12 \mathrm{~cm} / \mathrm{s}$. The aliasing velocity was $66-74 \mathrm{~cm} / \mathrm{s}$. In these settings the acquisition rate was 2130 frames/s.

A hand held rotation device (GE Vingmed Ultrasound) that was triggered by the $\mathrm{R}$ wave of the ECG rotated the transducer. To exclude cardiac arrhythmia, only heart cycles with a cycle length within $\pm 20 \%$ of the mean were accepted. The cardiac images were recorded during end expiratory apnoea within one breath hold, thereby abolishing the need for respiratory triggering. The six two dimensional images together with the ECG were stored in one cine-loop and then digitised and transferred on line to a computer (Apple, Cupertino, USA) for later data analysis. Total examination time including data storage was approximately 10-15 minutes depending on the acoustic window of the subject. 
ECHOCARDIOGRAPHIC DATA ANALYSIS

One of the authors (WYK) performed the entire echocardiographic analysis to reduce observer variation. This observer was blinded to the results of the exercise test and was unaware of any changes in the patient's symptoms at the time of analysis.

LV VOLUME ANALYSIS

The LV endocardial contours were drawn manually. In defining the endocardium, the papillary muscles were included in the LV volume to standardise measurements. The image at the beginning of the QRS of the ECG was defined as end diastole while the smallest area just before mitral valve opening was defined as end systole. Total LV volume was calculated from all six sections according to a volume estimation algorithm based on calculation of reconstructed polyhedrons-a set of polygons (usually triangles) that describe the object. ${ }^{13}$ The end diastolic (EDV) and end systolic (ESV) volumes were tabulated from the analysis and the ejection fraction (EF) was calculated as follows: $\mathrm{EF}=((\mathrm{EDV}-\mathrm{ESV}) / \mathrm{EDV}) \times$ $100 \%$ [equation 1].

The average of the two repeated recordings of EDV, ESV, and EF were tabulated. The analysis time for each LV volume was 4-5 minutes.

VOLUMETRIC FLOW ANALYSIS

The digitised colour Doppler flow maps were analysed with a dedicated software program. Stroke volumes (SVs) through the mitral valve (total SV) and LV outflow tract (forward SV) were calculated by integrating spatial and temporal colour flow velocity data. By integration of velocity vectors across a spherical surface, which is normal to the point of scanning, volumetric flow can be measured independently of the angle between the ultrasonic beam and the blood flow. In addition, phasic variations in the cross sectional flow area are included by summation of each colour Doppler frame. A detailed description of the 3D colour Doppler technique is provided elsewhere. ${ }^{9}{ }^{10}$ The mitral regurgitant fraction was calculated as follows: regurgitant fraction $=(($ total $\mathrm{SV}-$ forward $\mathrm{SV}) /$ total SV) $\times 100 \%$ [equation 2].

To characterise the mitral inflow waveform, the atrial systolic (A wave) contribution to mitral inflow was calculated as the ratio of the A wave time-velocity integral $\left(\mathrm{A}_{\mathrm{TVI}}\right)$ to the total diastolic time-velocity integral (Total $\left.{ }_{\mathrm{TVI}}\right)$. Thus, the atrial systolic contribution to mitral inflow was determined as $\mathrm{A}_{\mathrm{TVI}}$ Total $_{\mathrm{TVI}}$.

\section{EXERCISE PROTOCOL}

A six minute walk test was performed on the day before pacemaker implantation and 2-7 days after implantation to evaluate the effect of biventricular pacing on exercise capacity. Patients were instructed to walk down a corridor at their own pace, attempting to cover as much ground as possible in six minutes. The total walking distance was recorded.

STATISTICAL ANALYSIS

Paired Student's $t$ test was used to compare haemodynamic parameters measured by 3D echocardiography at baseline and during biventricular pacing. The 3D echocardiographic determinants of exercise capacity were explored by univariate and stepwise multiple linear regression analysis with the changes relative to baseline entered into the equation. All data are presented as mean (SD).

\section{Results}

FEASIBILITY

All patients had successful pacemaker implantation. The LV lead was inserted in a lateral or posterolateral vein in all but six patients, in whom the electrode was placed in an anterior vein. 3D echocardiography was feasible in all patients, but in one patient colour Doppler measurements were not available because of an error in data storage.

\section{VENTRICULAR RESPONSES TO BIVENTRICULAR} PACING

Biventricular pacing showed a variable haemodynamic response in different subjects resulting in large SDs for the patient group. The percentage changes for the $3 \mathrm{D}$ echocardiographic parameters - EDV, ESV, EF, forward SV, total SV, atrial systolic contribution to inflow, and mitral regurgitant fraction-are shown in table 2. Results of the six minute walk test and changes in QRS duration are shown in table 2. Pacing data were acquired with an atrioventricular interval of 113 (10) ms, which was significantly shorter than the baseline values of 240 (38) ms $(p<0.01)$. There were no significant differences between patients who were investigated one day after pacemaker implantation and patients investigated at baseline and 2-7 days after implantation.

Table 2 Summary of haemodynamic and exercise responses to biventricular pacing

\begin{tabular}{lllll}
\hline & Baseline & Pacing & \% Change & Palue \\
\hline HR (beats/min) & $79(14)(53-98)$ & $78(12)(60-98)$ & $-0.4(16)(-32$ to 30) & NS \\
EDV (ml) & $306(80)(223-512)$ & $294(77)(207-493)$ & $-4(5)(-15$ to 1$)$ & 0.01 \\
ESV (ml) & $230(81)(152-445)$ & $216(74)(142-418)$ & $-6(6)(-24$ to 2) & 0.02 \\
EF $(\%)$ & $26(6)(13-35)$ & $27(6)(15-35)$ & $1(3)(-2$ to 9$)$ & NS \\
FSV (ml) & $65(15)(45-106)$ & $73(13)(57-99)$ & $14(19)(-7$ to 56$)$ & 0.02 \\
TSV (ml) & $80(13)(64-118)$ & $78(9)(67-97)$ & $-1(15)(-23$ to 34$)$ & NS \\
A $_{\text {TVI } / \text { Total }}$ TVI $\left.\%\right)$ & $15(15)(0-46)$ & $27(10)(11-42)$ & $12(20)(-27$ to 41$)$ & 0.04 \\
MRF $(\%)$ & $20(13)(7-49)$ & $9(9)(0-30)$ & $-11(12)(-49$ to 9$)$ & 0.003 \\
6 min walk test (m) & $261(105)(0-390)$ & $378(82)(200-480)$ & $48(43)(11$ to 142$)$ & 0.00001 \\
QRS (ms) & $182(23)(145-240)$ & $150(16)(115-170)$ & $-16(10)(-29$ to 0$)$ & 0.00003
\end{tabular}

Values are mean (SD) with the maximum and minimum values in parentheses.

$\mathrm{A}_{\mathrm{TVI}} / \mathrm{Total}_{\mathrm{TVI}}$, atrial systolic contribution to diastolic inflow; EDV, end diastolic volume; ESV, end systolic volume; FSV, forward stroke volume measured by colour Doppler through the left ventricular outflow tract; MRF, mitral regurgitant fraction calculated according to equation 2 . NS, not significant; TSV, total stroke volume through the mitral valve. 


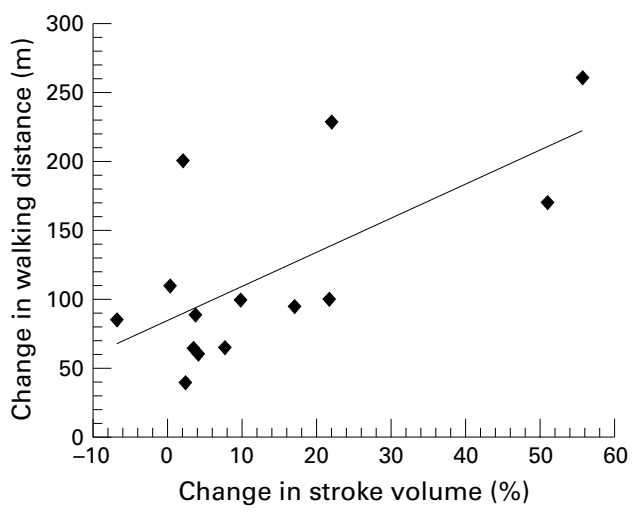

Figure 1 Correlation between percentage changes in forward stroke volume and increase in walking distance by six minute walk test. Patients with the largest increase in forward stroke volume had the greatest improvement in walking distance.

PREDICTORS OF CHANGES IN EXERCISE TEST Univariate 3D echocardiographic predictors of improved walking distance were the changes in forward SV, mitral regurgitant fraction, EDV, and ESV. Multiple linear regression analysis identified the percentage change in forward SV as the only independent factor determining absolute and percentage improvement in walking distance $\left(r^{2}=0.45 v r^{2}=0.73, \mathrm{p}=0.008 v\right.$ $\mathrm{p}=0.0002)$. One of the patients was not included in the analysis because of severe shortness of breath even at rest. In fig 1 the increase in walking distance is plotted versus percentage changes in forward SV. In five of 15 patients, an increase in forward SV above 15\% was seen with biventricular pacing and this was predictive of an improvement in walking

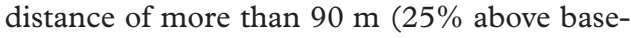
line).

INFLUENCE OF BASELINE QRS DURATION AND CHANGES IN QRS DURATION

Univariate regression analysis showed that the basal QRS duration predicted an increase in forward SV and a reduction in EDV and ESV

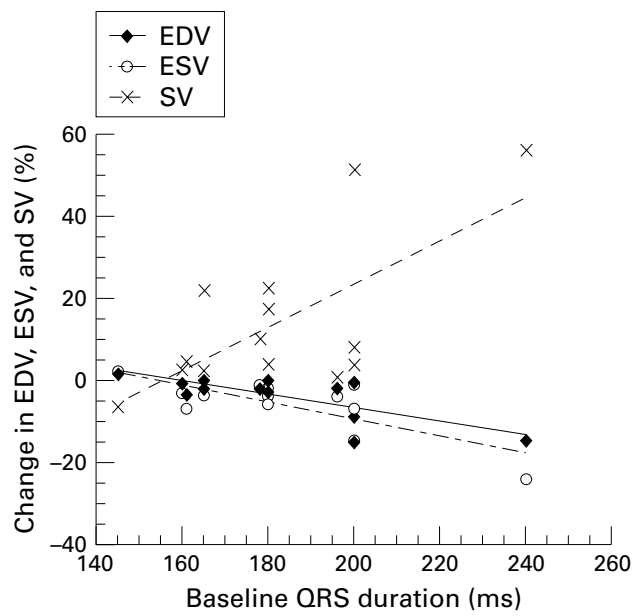

Figure 2 Correlation between baseline QRS duration and pacing induced changes in end diastolic volume (EDV), end systolic volume (ESV), and forward stroke volume (SV). Patients with the longest baseline $O R S$ duration showed the greatest benefit in terms of reduced left ventricular chamber size and increased forward stroke volume.

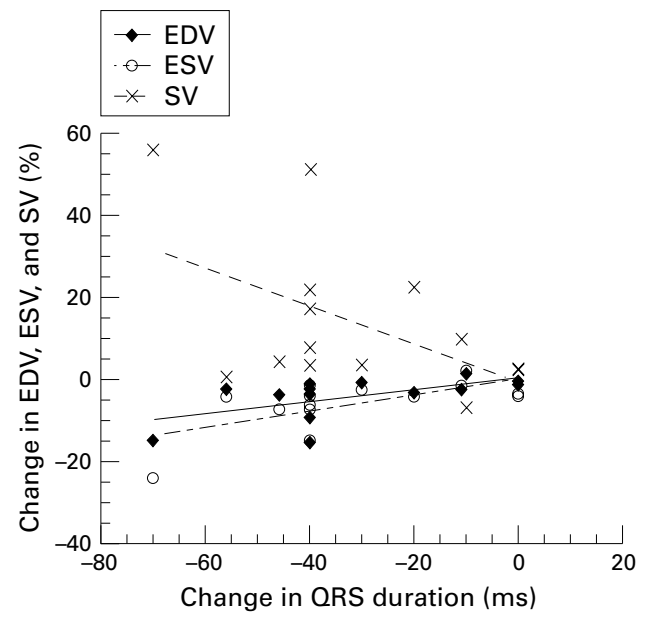

Figure 3 Correlation between pacing induced reduction in $Q R S$ duration and changes in end diastolic volume (EDV), end systolic volume (ESV), and forward stroke volume $(S V)$. Patients with the largest reduction in QRS duration showed the greatest haemodynamic benefit.

(fig 2). Thus, patients with baseline QRS duration less than $165 \mathrm{~ms}$ did not show any substantial haemodynamic benefit.

In addition, the reduction in QRS duration induced with biventricular pacing showed a modest but significant correlation with the increase in forward SV and the reduction in EDV and ESV (fig 3). Thus, patients with a reduction in QRS duration less than $20 \mathrm{~ms}$ did not show any substantial haemodynamic benefit. Stepwise multiple regression analysis revealed that baseline QRS duration was the only independent predictor of haemodynamic improvement in terms of increased forward SV and reduced EDV and ESV $\left(r^{2}=0.46-0.59\right.$, $\mathrm{p}<0.008$ to 0.001 ).

\section{Discussion}

The present study supports recent acute invasive haemodynamic data showing that biventricular pacing in patients with heart failure and ventricular conduction delay increases systolic pressure ${ }^{4}$ and cardiac index, ${ }^{5}$ and enhances LV contractility. ${ }^{6}$ Thus, with 3D echocardiography we showed that forward SV was significantly augmented with an immediate reduction in LV chamber size and mitral regurgitant fraction. The increase in forward SV was predictive of improved exercise capacity in the study group. This study therefore shows the potential of 3D echocardiography for evaluating the haemodynamic effects induced by biventricular pacing in patients with heart failure.

MECHANISMS OF HAEMODYNAMIC EFFECTS OF BIVENTRICULAR PACING

The only independent predictor of the short term improvement of exercise capacity was the increase in forward SV. This can be ascribed to the low output heart failure in these patients with predominantly systolic dysfunction. Ventricular SV is a function of preload, afterload, and contractility. ${ }^{14}$ Accordingly, SV increases when there is an increase in preload, a decrease in afterload, or augmented contractility. With 
respect to biventricular pacing, it is important to emphasise that the observed rise in forward SV seems to result from cardiac resynchronisation leading to increased $\mathrm{LV}$ contraction synchrony and reduced mitral regurgitation. Obviously, both mechanisms promote antegrade SV. The improved LV systolic function is shown in the reduced ESV, which depends on afterload and contractility but not on preload. ${ }^{14}$ It has been hypothesised that by specifically pacing the LV region with delayed electrical activation, contractile synchrony and thereby systolic function is partly restored leading to a reduction in ESV. This electromechanical hypothesis is strongly supported by our data, which showed that patients with the widest baseline QRS duration and the greatest pacing induced QRS narrowing had the greatest haemodynamic benefit. This seems logical in that the greater the magnitude of conduction delay (QRS duration), and thereby LV dyssynchrony, the more benefit resulted from resynchronisation.

Altered geometry of mitral leaflet attachments associated with LV dilatation is supposed to increase the tethering forces on the mitral valve apparatus restraining the leaflets from closing. In experimental models, functional mitral regurgitation has been shown to occur when papillary muscles are displaced in the posterior and mediolateral directions. ${ }^{15}$ Conversely, the reduction in ESV during biventricular pacing is likely to be responsible for the reduction in mitral regurgitant fraction because a reduced ESV decreased mitral valve tethering forces. Whether specific pacing sites such as the posterior or lateral LV walls are preferable to reduce mitral regurgitation has to be investigated in future studies. With reduced mitral regurgitation volume, volume unloading leads to reduced EDV, which explains why EF did not change despite a significant increase in SV.

Biventricular pacing changed the diastolic inflow pattern from one characterised primarily by an early filling wave to one with more physiologically balanced early and atrial filling components. However, we were not able to show a direct effect of this altered diastolic filling pattern on exercise capacity. In addition, there was no correlation to the changes in forward SV or LV chamber sizes. It is therefore not clear to what extent changes in mitral inflow pattern affect clinical outcome.

METHODOLOGICAL CONSIDERATIONS OF 3D ECHOCARDIOGRAPHY

Because 3D echocardiography was used to obtain all haemodynamic parameters including LV volumes and volumetric flow rates, the validity of these measurements deserves comment. The recent introduction of tissue harmonic imaging has drastically improved ultrasound image quality and especially the definition of endocardial borders, which is pertinent for LV volume measurements. Thus, 3D echocardiography with tissue harmonic imaging closely agreed with magnetic resonance imaging for assessment of LV volumes in both healthy subjects and patients with dilated cardiomyopathy. ${ }^{16}$ In addition, 3D echocardiography in comparison with two dimensional echocardiography showed excellent day to day reproducibility for LV volume measurements in healthy subjects. ${ }^{7}$ Thus, the reproducibility of two dimensional compared with 3D echocardiography with harmonic imaging, expressed as the $95 \%$ range for change, was $22 \% v 10 \%, 34 \% v 19 \%$, and $9 \%$ $v 5 \%$ for measurement of EDV, ESV, and EF, respectively. The reproducibility for the present study population should be similar since intraobserver variation (3-6\%), which had the greatest impact on total variance ${ }^{7}$, was similar in healthy subjects and in patients with severe LV dysfunction and dilated hearts. ${ }^{16}$ It has recently been shown that the choice of imaging method (two dimensional or 3D) has a greater impact on the results than does the choice of imaging modality (echocardiography or magnetic resonance imaging) when measuring LV volume and systolic function. ${ }^{17}$

The 3D colour Doppler method has previously been evaluated for quantification of volumetric flow in patients. ${ }^{11}$ Thus, since pacemakers are contraindicated for magnetic resonance imaging, 3D echocardiography is in our opinion the most reproducible non-invasive tool for quantitative haemodynamic evaluation of the effects of biventricular pacing. The advantage of 3D echocardiography over invasive protocols is the possibility for serial as well as long term studies to explore the more chronic effects of pacing.

\section{LIMITATIONS OF THE STUDY}

The changes in exercise capacity were evaluated by a six minute walking test, which in every case was performed at first during baseline condition and secondly after pacemaker implantation. It has been shown that familiarisation results in a small increase of $6 \%$ in walking distance between two successive tests performed one day apart. ${ }^{18}$ In addition, a placebo effect of the pacemaker implantation may be expected. The ideal situation would be to perform the exercise test in random order and blinded to both the patient and the observer. Thus, if we look conservatively at the improvement in walking distance and use a cutoff value, we may speculate that the 12 of 15 patients who showed a substantial improvement above $20 \%$ showed a genuine short term improvement in exercise capacity. A regular exercise test of maximal exercise capacity on a treadmill or bicycle would have been difficult to accomplish in the majority of the patients in this study population because of the severity of heart failure symptoms. Thus, the average walking distance during six minutes in the study population at baseline was only 261 (105) $\mathrm{m}$ (range 0-390 m).

The 3D echocardiographic analysis and, in particular, the manual tracing of LV volumes are subjective and depend on image quality. Thus, a totally blinded data analysis would be preferable; however, neither the visible leads in the right ventricle nor the pace spikes occurring 
in the ECG tracings permit this. Instead the observer was blinded to the results of the exercise tests, the clinical status of the patient, and all pacing related parameters. In addition, when the paired data for each subject were analysed, the results were not compared directly during the analyses. For volumetric flow analysis, subjectivity is much less of a problem because the colour Doppler flow maps explicitly define both the area and the velocity for each pixel, which are summarised to calculate flow.

In five of the patients, 3D echocardiography was not performed at baseline but one day after pacemaker implantation with biventricular pacing on or off. However, there were no significant differences in the haemodynamic response compared with the patients who were investigated at baseline and 2-7 days after implantation, indicating that the effect of pacing is rather acute.

PRACTICAL IMPLICATIONS

The variable haemodynamic response to biventricular pacing in consecutive patients with heart failure indicates that each patient's baseline haemodynamic values should be screened before pacemaker implantation to ensure a rational treatment strategy. Our data showed a significant reduction in mitral regurgitation in all patients with more than trivial regurgitation. Thus, the present study suggests that patients with intraventricular conduction delay and moderate to severe functional mitral regurgitation would benefit from biventricular pacing. We confirmed invasive haemodynamic observations that a wider baseline QRS is associated with greater mechanical improvement. ${ }^{6}{ }^{19} 20$ Our data also suggest that pacing induced changes in QRS width are predictive of the efficacy of biventricular pacing, even though the association was not very strong. In a six month follow up study, QRS duration during biventricular pacing was shown to be significantly shorter in patients who responded positively to pacing in terms of an increase in peak oxygen consumption rate. ${ }^{21}$ Other invasive studies did not show any predictive value of QRS narrowing but this could have been caused by differences in the pacing protocol, which in these studies was single site ventricular pacing ${ }^{60}$ compared with our simultaneous biventricular pacing protocol. LV single site pacing increased the width of the QRS complex. Therefore, at present baseline QRS duration remains an important parameter in both selection and evaluation of patients eligible for biventricular pacing. Tissue Doppler imaging may further improve patient selection by assessing the proportion of myocardium with asynchronous contraction. $^{22}$

\section{CONCLUSIONS}

3D echocardiography documented a significant short term haemodynamic improvement by biventricular pacing in patients with heart failure and intraventricular conduction delay. In five of 15 consecutive patients with heart failure, biventricular pacing induced a more than $15 \%$ increase in forward SV, which predicted a more than $25 \%$ increase in walking distance. A baseline QRS duration $\geqslant 165 \mathrm{~ms}$ with a reduction in QRS duration greater than $20 \mathrm{~ms}$ identified these patients. We believe that these results are very encouraging because they show that, in selected patients with medically refractory heart failure symptoms, biventricular pacing has the potential to provide a substantial and immediate improvement in haemodynamic profile and exercise capacity. Future studies should investigate the more chronic effects of biventricular pacing and try to establish even more precise criteria for patients who are candidates for pacing.

This work has been supported by grants from the Danish Heart Foundation, Copenhagen, Denmark.

1 Hochleitner M, Hortnagl $\mathrm{H}, \mathrm{Ng} \mathrm{CK}$, et al. Usefulness of physiologic dual-chamber pacing in drug-resistant idiopathic dilated cardiomyopathy. Am f Cardiol 1990;66:198202.

2 Brecker SJ, Xiao HB, Sparrow J, et al. Effects of dual-chamber pacing with short atrioventricular delay in dilated cardiomyopathy. Lancet 1992;340:1308-12.

3 Nishimura RA, Hayes DL, Holmes DR Jr, et al. Mechanism of hemodynamic improvement by dual-chamber pacing for severe left ventricular dysfunction: an acute Doppler and catheterization hemodynamic study. $7 \mathrm{Am}$ Coll Cardiol 1995;25:281-8.

4 Blanc JJ, Etienne Y, Gilard M, et al. Evaluation of different ventricular pacing sites in patients with severe heart failure: results of an acute hemodynamic study. Circulation 1997;96:3273-7.

5 Leclercq C, Cazeau S, Le Breton $\mathrm{H}$, et al. Acute hemodynamic effects of biventricular DDD pacing in patients with end-stage heart failure. $7 \mathrm{Am}$ Coll Cardiol patients with end-

6 Kass DA, Chen CH, Curry C, et al. Improved left ventricular mechanics from acute VDD pacing in patients with dilated cardiomyopathy and ventricular conduction delay. Circulation 1999;99:1567-73.

7 Kim WY, Søgaard P, Egeblad H, et al. Three-dimensional echocardiography with tissue harmonic imaging shows excellent reproducibility in assessment of left ventricular volumes. $\mathcal{F}$ Am Soc Echocardiogr (in press)

8 Sun Y, Ask P, Janerot-Sjoberg B, et al. Estimation of volume flow rate by surface integration of velocity vectors from color Doppler images. F Am Soc Echocardiogr 1995;8:90414.

9 Kim WY, Poulsen JK, Terp K, et al. A new Doppler method for quantification of volumetric flow: in vivo valimethod for quantification of volumetric flow: in vivo vali-
dation using color Doppler. $\mathcal{F}$ Am Coll Cardiol 1996;27: dation $182-92$.

10 Poulsen JK, Kim WY. Measurement of volumetric flow with no angle correction using multiplanar pulsed Doppler ultrasound. IEEE Trans Biomed Eng 1996;43: 589-99.

11 Kim WY, Poulsen JK, Terp K, et al. New semi-automated Doppler method for quantification of volumetric flow. Intraoperative validation using multiplane transesophageal color Doppler imaging. I Am Soc Echocardiogr 1997;10: 330-6.

12 Cruz-Orive LM, Roberts N. Unbiased volume estimation with coaxial sections: an application to the human bladder. 7 Microsc 1993;170:25-33.

13 Gilja OH, Smievoll AI, Thune N, et al. In vivo comparison of 3D ultrasonography and magnetic resonance imaging in volume estimation of human kidneys. Ultrasound Med Biol 1995;21:25-32.

14 Frankel SK, Fifer MA. Heart failure. In: Lilly LS, ed. Pathophysiology of heart disease. Baltimore, Williams and Wilkins, 1998:200

15 Otsuji Y, Handschumacher MD, Schwammenthal E, et al. Insights from three-dimensional echocardiography into the mechanism of functional mitral regurgitation: direct in vivo demonstration of altered leaflet tethering geometry. Circulation 1997;96:1999-2008.

16 Kim WY, Søgaard P, Kristensen BØ, et al. Measurement of left ventricular volumes by three-dimensional echocardiography with tissue harmonic imaging. A comparison with magnetic resonance imaging. $\mathcal{F} \mathrm{Am}$ Soc Echocardiogr (in press)

17 Chuang ML, Hibberd MG, Salton CJ, et al. Importance of imaging method over imaging modality in noninvasive determination of left ventricular volumes and ejection cardiography and magnetic resonance imaging. 7 Am Coll Cardiog 2000;35:477-84.

18 Refsgaard J. Neurohormonal, metabolic and cardio-vascular aspects of treatment with a vasodilating beta-blocker in aspects of treatment with a vasodilating beta-blocker in
patients with congestive heart failure [dissertation]. Aarhus: Aarhus University, 2000:64. 
19 Auricchio A, Stellbrink C, Block M, et al. Effect of pacing chamber and atrioventricular delay on acute systolic function of paced patients with congestive heart failure. The pacing therapies for congestive heart failure study group. The guidant congestive heart failure research group. Circulation 1999;99:2993-300

20 Nelson GS, Curry CW, Wyman BT, et al. Predictors of systolic augmentation from left ventricular preexcitation in patients with dilated cardiomyopathy and intraventricular conduction delay. Circulation 2000;101:2703-9.
21 Alonso C, Leclercq C, Victor F, et al. Electrocardiographic predictive factors of long-term clinical improvement with multisite biventricular pacing in advanced heart failure. $\mathrm{Am}$ f Cardiol 1999;84:1417-21.

22 Søgaard P, Kim WY, Mortensen PT, et al. Impact of biventricular pacing on left ventricular remodeling and performance in patients with severe heart failure. A threedimensional echocardiographic and tissue Doppler study [abstract]. Eur Heart $\mathcal{F}$ 2000;21:410.

\section{IMAGES IN CARDIOLOGY}

\section{Acute thrombotic occlusion of the right coronary artery treated successfully with a helical thrombectomy device}

In these figures we illustrate the successful removal of visible occlusive thrombus from the right coronary artery with a new helical thrombectomy device (X-Sizer).

A 40 year old male smoker with known but untreated hyperlipidaemia was admitted with
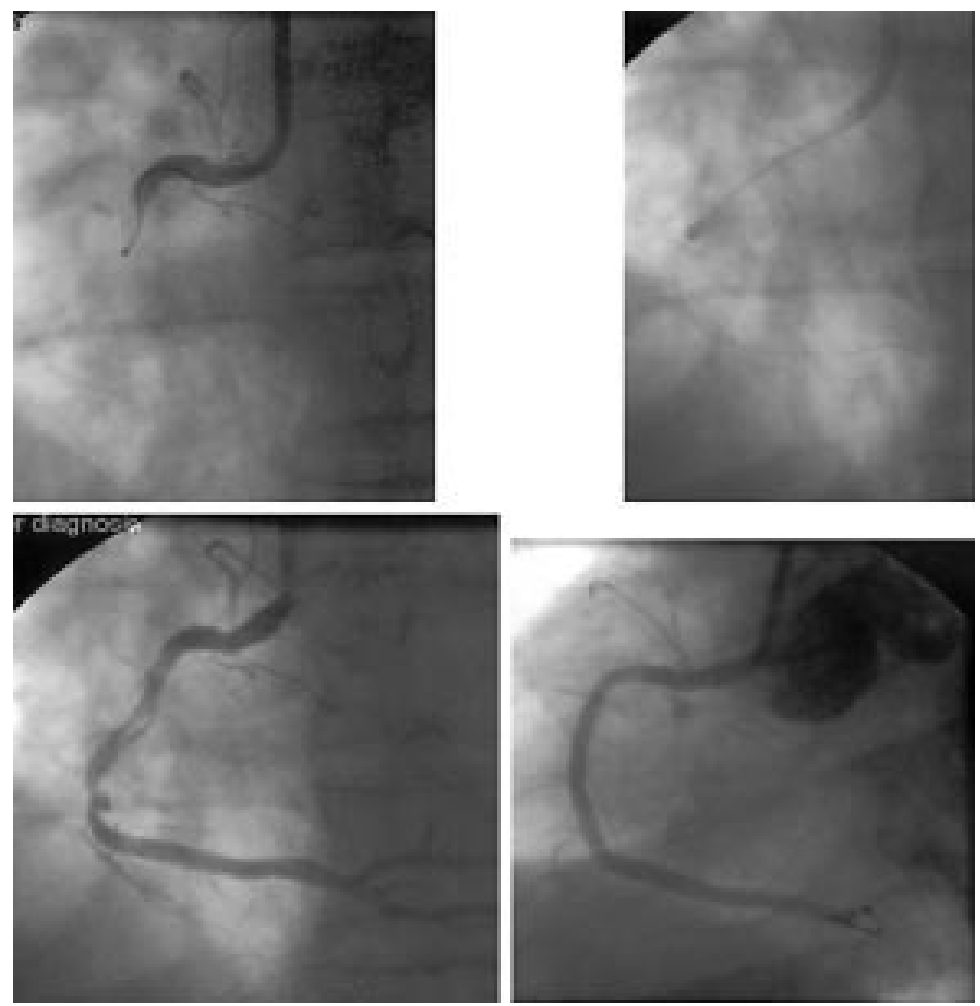

an acute inferior myocardial infarction. Despite treatment with thrombolysis within two hours of onset of his symptoms he continued to experience chest pain and ECG ST segment elevation failed to improve. He was transferred to our hospital and angiography at approximately three hours post-thrombolysis showed a total acute occlusion of the culprit vessel. Despite crossing the lesion with a guide wire there was no distal flow. The X-Sizer was advanced over the wire and activated with a hand held, battery operated source to achieve restoration of TIMI III flow. Histology of the excised fragments showed fresh acellular thrombus. The procedure was completed with the deployment of two stents and no acute complication. No glycoprotein IIb/IIIa antagonist was used.

Thrombosis in the background of plaque rupture or fissure is increasingly recognised to be the pivotal event in the pathogenesis of acute coronary syndromes, not only in the acute myocardial infarction but also in the syndrome of unstable angina/non- $\mathrm{Q}$ wave myocardial infarction. While the outcome after percutaneous intervention is normally good, the presence of visible thrombus is associated with a worse outcome and more complications. Until recently, there has been no safe and effective means of removing thrombus mechanically before balloon angioplasty and stenting.

This is a very promising new device for a common clinical problem.

S CONSTANTINIDES

R JONES

M F SHIU

savvas@sconstantinides.freeserve.co.uk 